\title{
Testing the optical methods by using the multi-level holographic grating
}

\author{
O.I. Barchuk ${ }^{1}$, Y.V. Braginets, O.S. Klimov, Y.A. Oberemok ${ }^{2}$, S.N. Savenkov \\ Taras Shevchenko Kyiv National University, Radiophysics Department, \\ 5 build., 2, Acad. Glushkov ave., 03127 Kyiv, Ukraine, \\ phone: (380-44)526-04-83; e-mail1' : boi@univ.kiev.ua, e-mail' : fix@univ.kiev.ua
}

\begin{abstract}
In this work the interaction peculiarities of electro-magnetic optical range radiation with gratings' surfaces are investigated. The multilevel diffractive holographic grating is proposed to be used for the polarization optical methods testing. This object allowed to obtain simultaneous visualization of different spatial frequencies and to estimate both structure and surface peculiarities when working with 3D-objects. Using this additional information one can remove uncertainty in solution of the inverse problem of ellipsometry related with ellipsometric angles periodicity. Thereby, multiangle ellipsometry allowing investigation of the specular reflection component could be used to study submicron peculiarities of the object. We have also presented the basic aspects of ellipsometric method optimization. It was shown that anisotropy parameters, such as linear amplitude anisotropy and linear phase anisotropy, obtained from ellipsometric measurements are the most effective to ascertain the submicron characteristic dimension of material.
\end{abstract}

Keywords: diffraction, holographic grating, submicron heterogeneity, ellipsometry.

Manuscript received 09.12.08; accepted for publication 18.12.08; published online 02.03.09.

\section{Introduction}

A lot of optical experimental methods based on interaction of electromagnetic waves with medium possess high sensitivity even at a submicron characteristic dimension of the material under investigation. [1-3]. However, their usage is often complicated by the necessity of reliable interpretation of the experimental data.

The diffraction gratings are widely used as test samples for calibration of different optical methods [37]. Main advantage of grating application seems to be the strongly pronounced character of collective interaction between formations (clusters) with a welldefined characteristic dimension that corresponds to the lattice parameter. If taking into account availability of nano-structural materials with the characteristic dimension comparable to the wavelength of optical radiation $\lambda$, the use of diffraction gratings for testing requires additional researches.

Really, according to the diffraction grating theory the well-known relation for angles $\varphi$ that indicate the direction of the diffraction maxima $\sin \varphi=m \lambda / d_{1}\left(d_{1}-\right.$ lattice parameter, $m$ - number of a diffraction maximum) for the nanometer value $d_{1}$ becomes invalid because of $\sin \varphi$ reaches values $\geq 1$. In such a case, diffraction orders are located nearby the grating surface or dissapear at all. The aforementioned ratio is correct, if we would use parallel sounding beams. It is clear that visualization of diffraction orders from nanometer-size grating periods needs to use a special optical setup.

For example, in the works [3, 4] authors used the optical scheme with oblique incidence of the light beam, and the measured diffraction efficiency after reflection from the grating depended on polarization. A similar polarization dependence in diffraction orders were observed by the authors [5-9]. Thus, the multi-angle ellipsometry is an informative method that could be used in the needed range for angles of incidence and angles of scattering. It was possible to investigate a specular reflection component suitable for experiments with unknown objects.

In connection with above-stated, the aim of this paper was to create a universal special grating with periods equal to $\lambda / 10 \leq d_{i} \leq 10 \lambda$, the optical scheme and measuring methods suitable for visualization and investigation of polarization characteristics. 


\section{Ellipsometric investigation features for ordinary grating}

It was earlier shown that the multiangle ellipsometry can be used to study the surface structures and subsurface formations with submicron and nanometer dimensions $[6,9,10]$.

The experiment contains the study how the structure of the samples affects on the behavior of reflected light polarization under various angles of incidence. To reach that purpose, we used the Mueller polarimeter based on the modified three-input polarization method.

The polarimeter (Fig. 1) consists of two channels: the probing and receiving ones. There are He-Ne laser with the wavelength $\lambda=0.63 \mu \mathrm{m}$ and polarizer, orientation $\theta$ of which is controlled by a computer in the probing channel. The receiving channel includes the rotating quarter-wave plate and polarizer (analyzer).

According to [11], this modification of the polarimeter allows to measure the first three columns of the Mueller matrix simpler and with a higher precision. Thus, the mentioned part of the Mueller matrix is quite enough to complete description of polarization properties for the object with weakly pronounced depolarization [12-14].

We have realized three experiments when Mueller matrices for the chosen sample were measured by three optimized methods: 1) four-input polarization method (FIPM) [15], 2) three-input polarization method with a circular polarized source $\left(\mathrm{TIPM}_{\mathrm{cir}}\right)$ [14], and 3) threeinput polarization method with a linear polarized source $\left(\mathrm{TIPM}_{\text {lin }}\right)$ [11]. Standard deviations (SD) for the Mueller matrix elements measured using the above mentioned methods for 6,000 averaging at the angle of incidence $56^{\circ}$ are presented in Table 1 . In this case, the matrix of averaged values was:

$\overline{\mathbf{M}}_{4 \times 3}=\left[\begin{array}{ccc}1.0000 & 0.2060 & 0.0100 \\ 0.1900 & 0.9868 & 0.1014 \\ 0.0108 & 0.1039 & -0.9029 \\ 0.0008 & 0.0121 & -0.3295\end{array}\right]$

The anisotropy parameters were considerable at this angle of incidence. Therefore, the error of measurements had to reach its maximum.

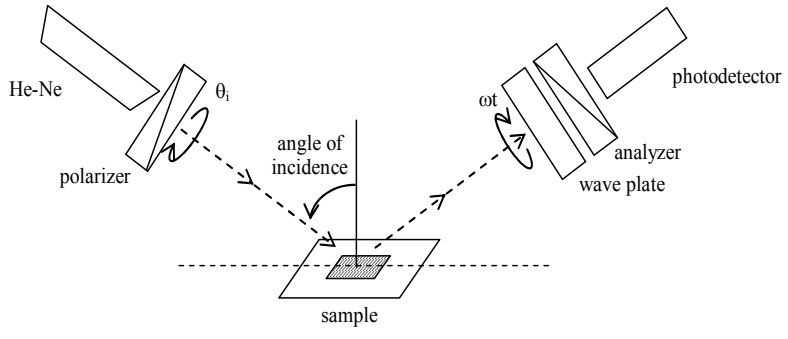

Fig. 1. Setup for multi-angle Mueller polarimetry.

As it is seen from Table 1, TIPM ${ }_{\text {cir }}$ method has the highest stability, which was followed by TIPM $\mathrm{M}_{\text {lin }}$ and then FIPM (with forming $\lambda / 4$-plate), as it was expected $[11,15]$. However, we have chosen TIPM $_{\text {lin }}$ for usability [11].

The measured Mueller matrices were analyzed within the framework of the multiplicative matrix model developed in [15-17]. This model is based on the assumption that any complex elastic interaction of polarized radiation with an object can be described by involving four basic types of anisotropy. They are linear phase (LinPh) and amplitude (LinAm) anisotropy as well as circular phase ( $\mathrm{CirPh})$ and amplitude (CirAm) anisotropy.

Table 2 demonstrates the calculated standard deviation for anisotropy parameters that corresponds to the mean values $\bar{R}=0.000, \bar{P}=0.670, \bar{\gamma}=1.40^{\circ}$, $\bar{\delta}=161.47, \bar{\alpha}=1.45, \bar{\phi}=-0.17^{\circ}$ depending on the method of measurements.

The values $R$ and $\phi$ are circular amplitude and phase anisotropy, correspondingly; $P, \delta$ are values of linear amplitude and phase anisotropy, correspondingly; $\gamma$ and $\alpha$ are orientation of linear amplitude and phase anisotropy, respectively.

Data presented in Table 1 corroborate experimentally the estimations of the Mueller matrix measurement errors derived theoretically in $[11,15]$ for FIPM, TIPMlin and TIPMcir. However, it is substantial that Table 2 shows that this is not the case for the errors of determination of the values for some anisotropy parameters (see, for example, the value of linear phase anisotropy $\delta$ ). This can be explained by the peculiarities

Table 1. Standard deviations for Mueller matrix elements that were measured using various ellipsometric methods.

\begin{tabular}{|c|c|c|c|c|c|c|c|c|c|c|c|c|}
\hline Method & $\mathrm{m}_{11}$ & $\mathrm{~m}_{12}$ & $\mathrm{~m}_{13}$ & $\mathrm{~m}_{21}$ & $\mathrm{~m}_{22}$ & $\mathrm{~m}_{23}$ & $\mathrm{~m}_{31}$ & $\mathrm{~m}_{32}$ & $\mathrm{~m}_{33}$ & $\mathrm{~m}_{41}$ & $\mathrm{~m}_{42}$ & $\mathrm{~m}_{43}$ \\
\hline FIPM & 0.38 & 0.49 & 0.47 & 0.16 & 0.56 & 0.68 & 0.32 & 0.62 & 0.63 & 0.24 & 0.45 & 0.39 \\
\hline $\mathrm{TIPM}_{\text {lin }}$ & 0.13 & 0.24 & 0.35 & 0.29 & 0.52 & 0.30 & 0.39 & 0.53 & 0.13 & 0.13 & 0.17 & 0.04 \\
\hline $\mathrm{TIPM}_{\text {cir }}$ & 0.06 & 0.20 & 0.16 & 0.20 & 0.30 & 0.41 & 0.22 & 0.31 & 0.32 & 0.07 & 0.09 & 0.07 \\
\hline
\end{tabular}



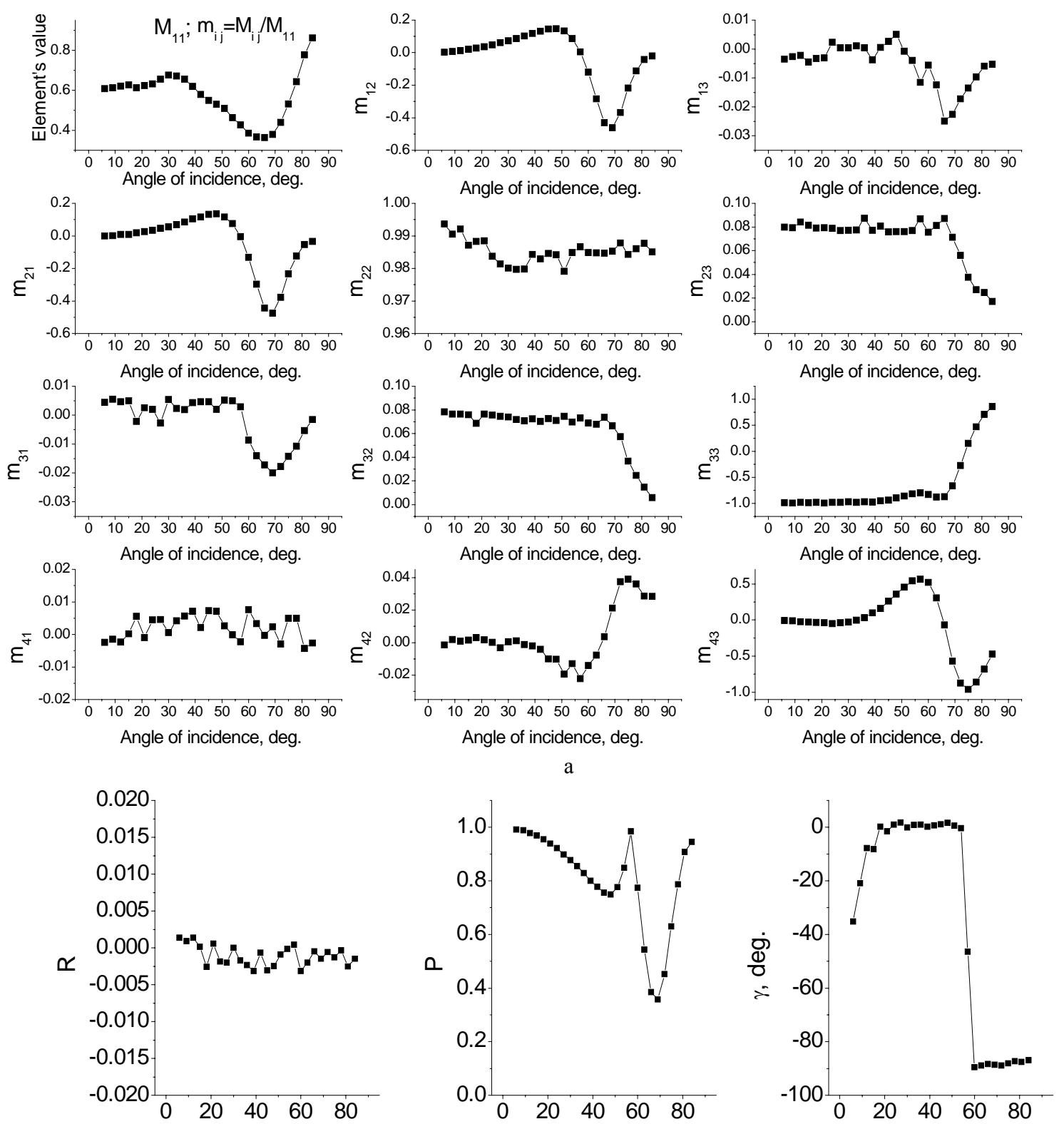

Angle of incidence, deg.
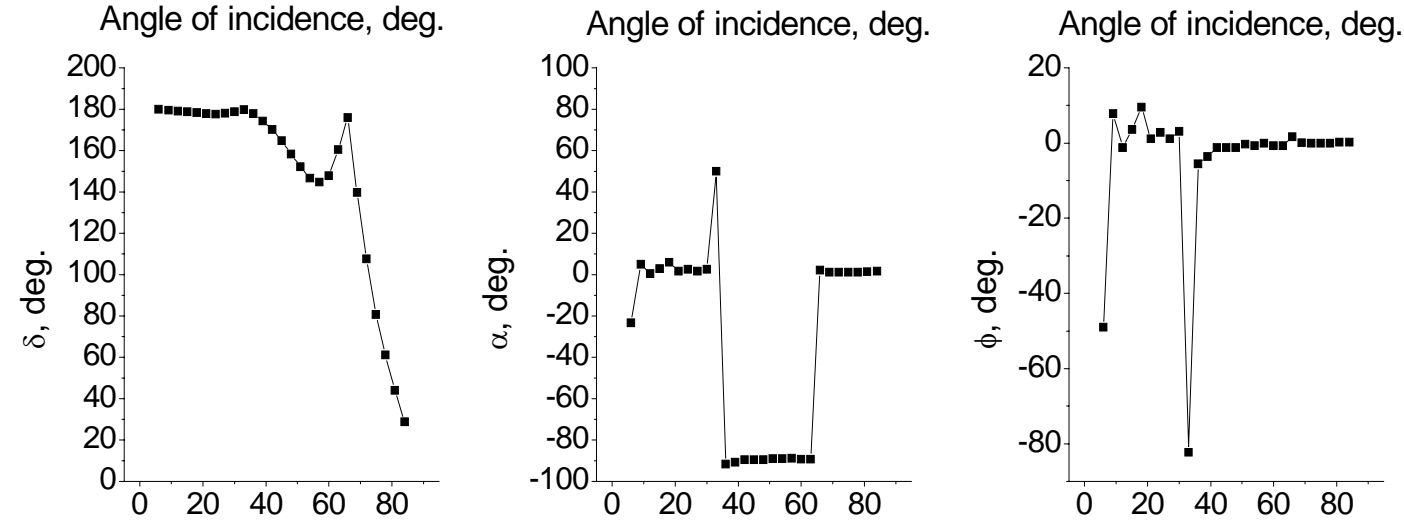

Angle of incidence, deg.

Angle of incidence, deg.

Angle of incidence, deg.

Fig. 2. Dependences of Mueller matrix elements (a) and anisotropy parameters (b) on the angle of incidence for the sample with no grating structure. 
Table 2. Standard deviations for anisotropy parameters that were calculated for various ellipsometric methods.

\begin{tabular}{|c|c|c|c|c|c|c|}
\hline $\begin{array}{c}\text { SD } \\
\text { Method }\end{array}$ & $R$ & $P$ & $\begin{array}{c}\gamma, \\
\text { deg }\end{array}$ & $\begin{array}{c}\delta, \\
\text { deg }\end{array}$ & $\begin{array}{c}\alpha, \\
\text { deg }\end{array}$ & $\begin{array}{c}\phi, \\
\text { deg }\end{array}$ \\
\hline FIPM & 0.0012 & 0.004 & 0.43 & 0.47 & 0.17 & 0.21 \\
\hline TIPM $_{\text {lin }}$ & 0.0005 & 0.003 & 0.29 & 0.08 & 0.04 & 0.08 \\
\hline TIPM $_{\text {cir }}$ & 0.0002 & 0.001 & 0.51 & 0.11 & 0.02 & 0.10 \\
\hline
\end{tabular}

of individual measurement error distributions for matrix elements $\Delta m_{i j}$, which are presented in Table 2. Thus, individual errors of the matrix element measurements give additional important information on stability of the inverse problem solution.

Thus, for each sample the Mueller matrix was measured and anisotropy parameters were calculated at various incidence of light. Corresponding dependences for a sample with no grating structure are presented in Fig. 2 and demonstrate qualitative agreement of Mueller matrix elements for similar investigations [6].

The dependences in Fig. 2 are reference lines and predicted localization of extrema on these dependences for a sample with no grating structure.

To investigate objects that have a characteristic dimension (both relief height and lateral size) less than the wavelength of electromagnetic sounding, we prepared the gratings with the identical periods $d$ ( $d=$ $1 \mu \mathrm{m})$ and variable height $h_{i}, i=1-25$. The gratings were produced by e-beam lithography and were described in more details in the following section.

In the paper [9], we saw that diffraction maximum positions were unaltered at the quasi-normal angle of incidence $\left(10^{\circ}\right)$, but their intensities depended on diffraction grating groove depths for the main and first orders (Fig. 3). The behavior of those dependences shows their anti-correlation and illustrates the energy redistribution between diffraction maxima (from the main to higher orders).

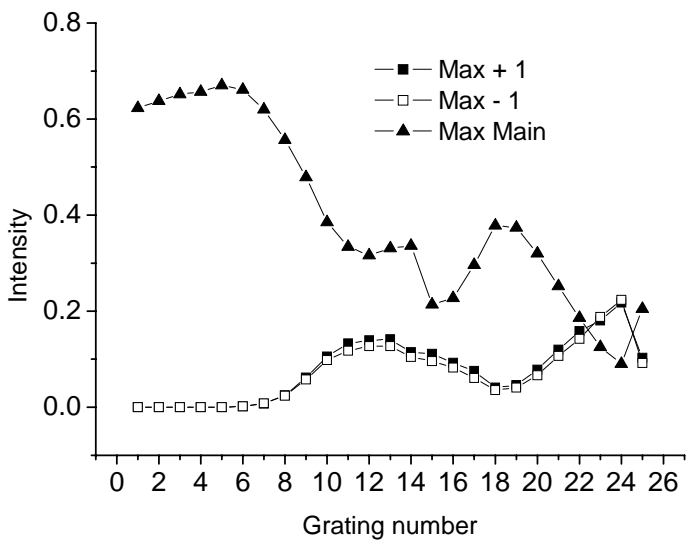

Fig. 3. Intensities of the main and first diffraction orders for various numbers of gratings 1-25 (which correspond to $h=12$ $300 \mathrm{~nm})$.
Angular dependences of the orthogonal component of the reflection coefficients were different for gratings with different heights $h_{i}$ and for different polarization of sounding illumination [9]. Using the method TIPM $_{\text {lin }}$, we studied 25 gratings with different depths. Only for the first 11 ones good repeatability was shown for the measured and calculated data.

It is significant that parameters $P$ and $\delta$ demonstrate the heighten sensibility to surface changes (in what following, we will consider the only $P$ and $\delta$ ). Below, the selected dependences versus the angle of incidence for a linear amplitude $(P$-parameter) and linear phase anisotropy ( $\delta$-parameter) for gratings with the depth step $12 \mathrm{~nm}$ are shown in Fig. 4.

It is seen that initial extrema move to the range of less angles of incidence with a rising grating depth. In addition, the values of extrema become narrower, and new maxima (for $P$ ) appear.
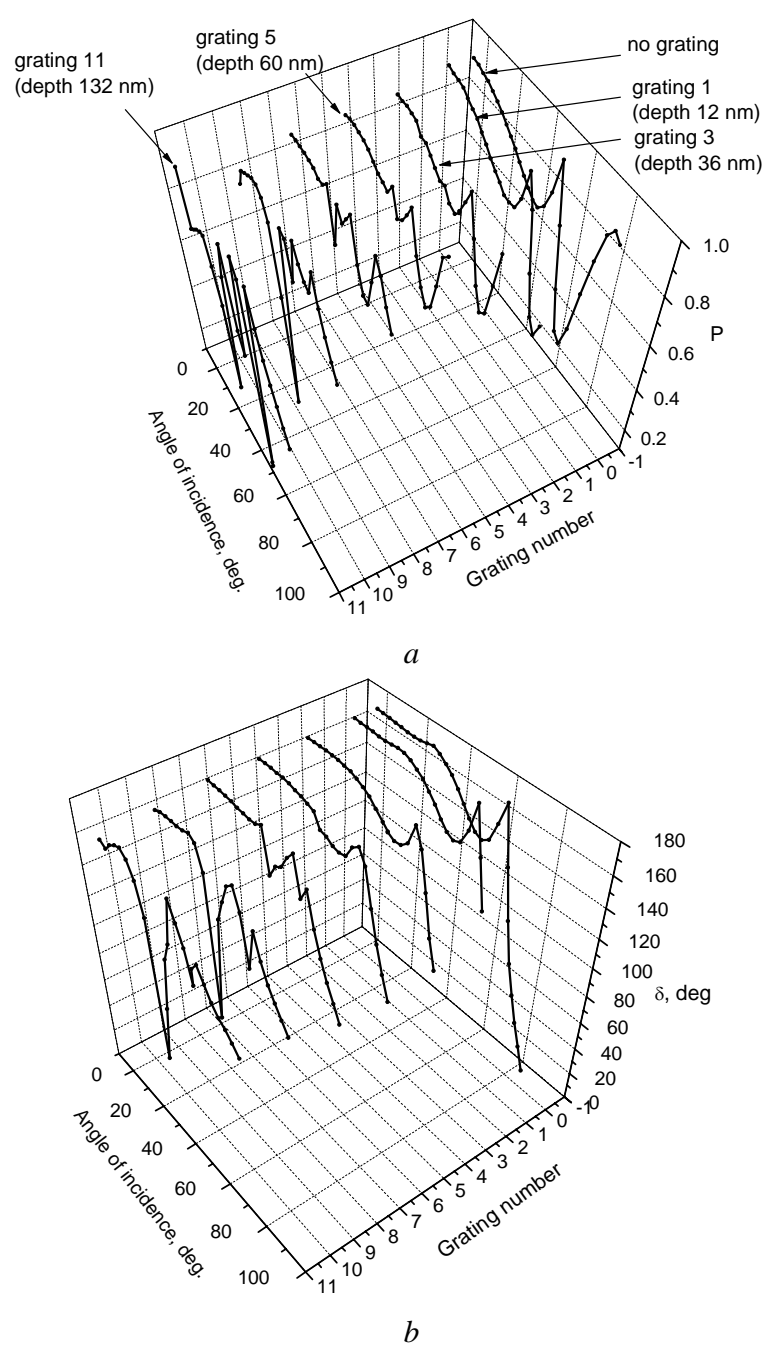

Fig. 4. Angular dependences of the linear amplitude anisotropy $P$ (a) and linear phase anisotropy $\delta$ (b) for gratings with numbers: $1,3,5,7,9,11$. 


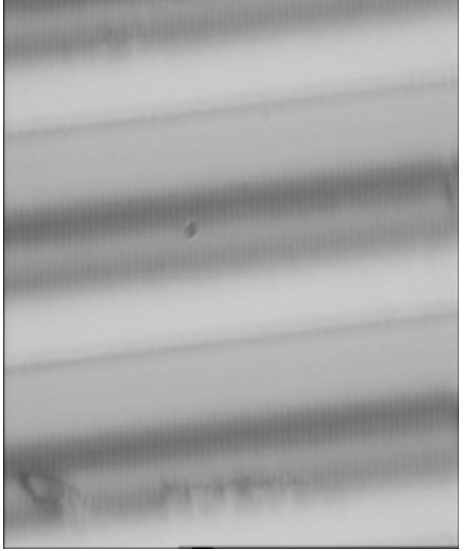

(a)

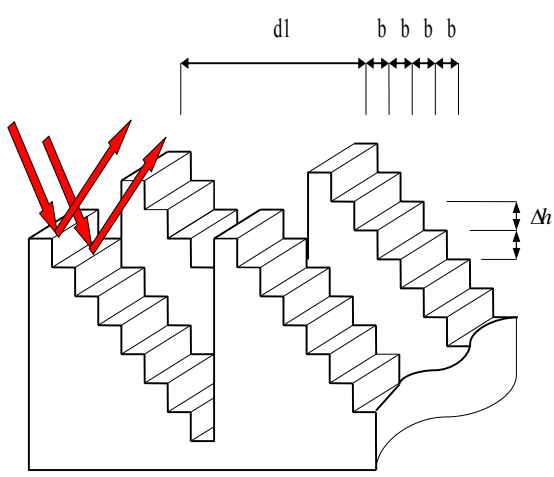

(b)

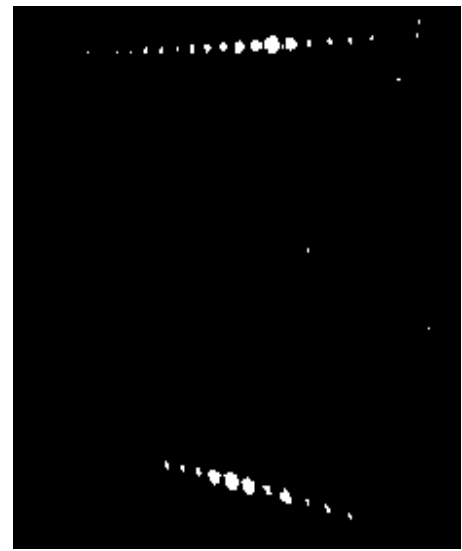

(c)

Fig. 5. Images of the multi-level diffractive holographic grating obtained using optical microscopy (a), its schematic sketch (b), simultaneous visualization of lines with the main and +1 st diffraction orders from this grating (c).

\section{The multi-level diffractive holographic grating}

We cannot predict existence of clusters with different heights and characteristic dimension, their quantity and their location on the surface under investigation of unknown objects. Therefore, creation of the test with well-defined surface, with needed parameters both along the surface and perpendicular to it, with known anisotropic properties, is a very important and urgent problem. However, such test based on the structure with a grating region, according to [7] could lead to excitation of several modes, and the energy of the incident wave is distributed into diffraction orders at the certain necessary conditions. This fact needs to take into account the grating design.

In this paper, we proposed the technical decision to create diffraction gratings that have to meet the following requirements:

- to conclude needed different spatial frequencies selected by the height levels (multi-level grating);

- it should not have some additional modes caused by grating range that could be reached by special relief shape;

- it should not have symmetry for simultaneous visualization of both low and deep levels for high spatial frequencies.

All grating objects, which are the subject under consideration in this paper, were produced by e-beam lithography on electronic-sensitive PMMA resist $\left(n_{1}=1.515, \quad \kappa_{1}=0.0019\right)$ with the thickness equal to $642 \mathrm{~nm}$. PMMA resist was deposited on $\mathrm{Cr}$ layer $\left(n_{2}=3.48, \quad \kappa_{2}=4.36\right)$ with the thickness equal to 2 $\mu \mathrm{m}$, which covered a glass substrate $\left(n_{3}=1.5\right)$ with the thickness equal to $2 \mathrm{~mm}$. Indicated in brackets are the refractive $n_{j}$ and the absorption $\kappa_{j}$ indices for corresponding layers.
Also, the multi-level diffractive holographic grating produced by e-beam lithography according to the scanning program for e-beam proposed to be used for testing the polarization optical methods. There are 64 quantization height levels (from 4.69 to $300 \mathrm{~nm}$ ) and corresponding periods (from 0.4 to $25.6 \mu \mathrm{m}$ ) in one direction. The grating period is constant $(1.2 \mu \mathrm{m})$ on another perpendicular direction. Thus, such grating represents set of echelette grating in one direction and ordinary grating with gaps width equal to $0.4 \mu \mathrm{m}$ and strips width equal to $0.8 \mu \mathrm{m}$ in another perpendicular direction.

There is imaging of multi-level diffractive holographic grating by optical microscopy (a), its schematic sketch (b), simultaneous visualization of lines with the main and +1 st diffraction orders from this grating (c) in Fig. 6. The obtained diffraction pattern from this holographic grating was a composite one and consisted of lines with diffraction maxima of various intensities and periods in two orthogonal directions.

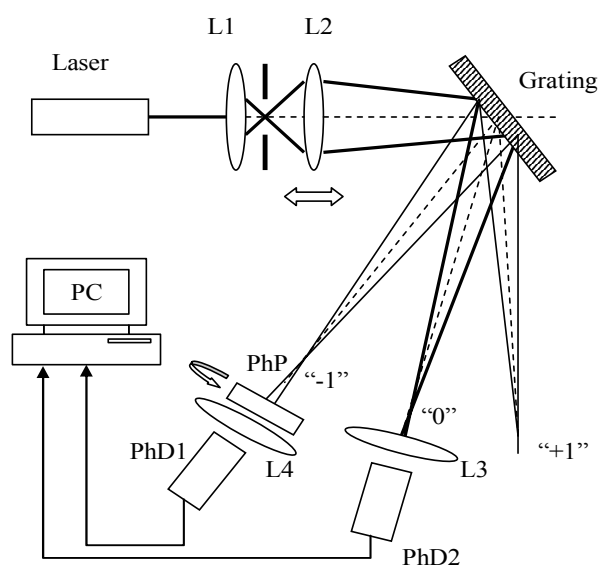

Fig. 6. Setup for simultaneous visualization of different spatial frequencies. 
In this case, simultaneous visualization of different spatial frequencies on one screen is problematic, because of system geometry is not paraxial. Focusing the diffraction orders occurred on a spherical surface caused by the goniometry application. Diffraction maxima have different intensities, which relates with a phase difference of interfering beams in this direction. We saw good diffraction maximum spacing that allowed to receive the good angular resolution down to $0.05^{\circ}$. Depending on investigation object parameters, application of this grating as a test object can be possible in various configurations of the optical setup.

We proposed converging beams for visualization of spatial frequencies corresponding to submicron changes on the surface (Fig. 6).

As an illumination source, He-Ne laser with $\lambda=0.63 \mu \mathrm{m}$ was used. The coherent radiation was formed into converging beams by the long-focus system $\mathrm{L}_{1} \mathrm{~L}_{2}$ and illuminated the grating entirely. After reflection from the grating, the main maximum in the specular reflection direction and a great number of secondary orders were observed. Application of a convertible lens enabled suitable observation orders from a submicron dimension that were upright spacing from zero order.

Diffraction maxima corresponding to characteristic dimension more than $\lambda$ were situated across from the central maximum on a short distance from each other. To have effective restoration of every maximum, we used the lens $\mathrm{L}_{3}$ that could enlarge image to a needed scale. The photodetectors $\mathrm{PhD}_{1}$ and $\mathrm{PhD}_{2}$ were intended to register the diffraction maximum intensities and were situated on the goniometry arms. There is $\mathrm{PhD}_{1}$ with lens $\mathrm{L}_{4}$ and calibrated phase plate $\mathrm{PhP}$ in the channel for investigation submicron heterogeneities. To reach more accurate investigation for high spatial frequencies, CCDcamera with subsequent computer data processing instead of $\mathrm{PhD}_{1}$ can be used.

We could achieve such location of diffraction orders, when specular reflection component is fixed only at zero position of the scale reading (for goniometry) at a corresponding slope of grating. In this case, intensities of maxima could be measured only in the channel for investigation submicron heterogeneities by CCD-camera and $\mathrm{PhP}$.

Comparisons with objects that have nano- and submicron peculiarities give a chance to estimate range of spacing frequencies for an unknown object. Thereby, we can remove uncertainty in solution of the inverse ellipsometric problem related with periodicity of ellipsometric angles [18].

A substantial moment, when investigating unknown samples are positioning of their surfaces like to multi-level grating.

Then, the ellipsometric measurements of this object in the specular direction (zeroth order) were produced (Fig. 7).
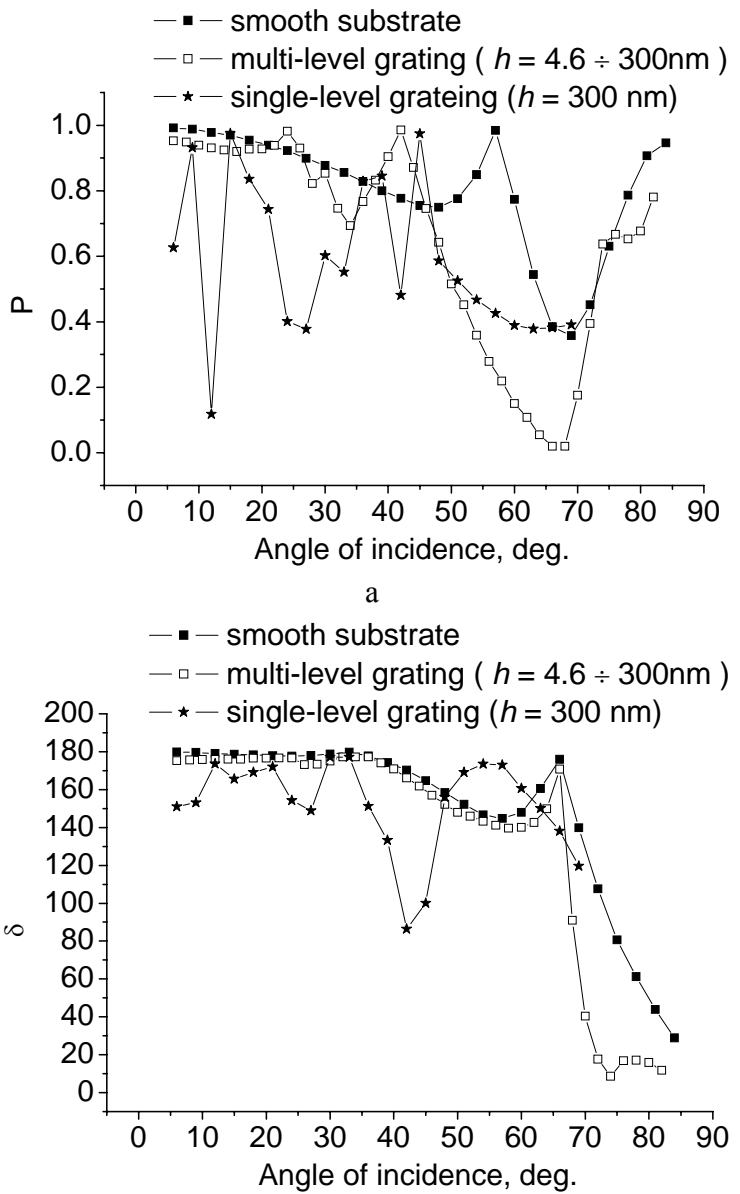

$b$

Fig. 7. Angular dependences of the linear amplitude anisotropy $P$ (a) and linear phase anisotropy $\delta$ (b) for objects without any grating structure (filled squares), multi-level holographic grating (open squares), ordinary grating with the number 25 corresponding to $h=300 \mathrm{~nm}$ (asterisks).

The dependences of anisotropy parameters $P$ and $\delta$ were compared with analogous results for clear substrate (without grating range, Fig. 2b). Their behaviors are similar and compatible units at certain scaling. Absence of additional extrema in dependences for the multi-level grating can be related with absence of the propagating mode.

In that way, these facts can be added to advantages of multi-angle-incidence ellipsometry using the specular component, because all changes on the surface can be reflected in polarization properties of objects (even excitation of the propagating mode).

\section{Conclusion}

The obtained results show that structural submicron dimension changes on surface of different samples can be investigated in the optical range by multi-angleincidence ellipsometry. As a test object, the periodical structure with well-defined parameters can be used. In 
our case, such object was multi-level holographic grating. This object was produced in the layer of PMMA photoresist. This method may preset the required values of the groove height and shape with the lateral resolution $0.4 \mu \mathrm{m}$ and depth of the step $12 \mathrm{~nm}$. However, there is some critical dimension after which incident illumination leads to appearance of the propagating mode in ordinary gratings (relief of the "meander" type) [7]. This fact complicates character of interaction with structured surface and leads to ambiguities when analyzing their parameters by using polarimetric methods. Expansion of dynamic range testing for test object with characteristics that are more than the mentioned critical dimension need a more complicated shape that excepts excitation of the lateral mode. In this work, the proposed multi-level grating with a broad bandwidth of heterogeneity dimensions and corresponding to them spatial frequencies was used as this test object.

\section{References}

1. L.A. Golovan, V.Yu. Timoshenko, P.K. Kashkarov, Optical properties of porous-system based nanocomposites // Uspekhi Fizicheskikh Nauk 177(6), p. 619-638 (2007) (in Russian).

2. F. Lagugne' Labartheta, P. Rochon, A. Natansohn, Polarization analysis of diffracted orders from a birefringence grating recorded on azobenzene-containing polymer // Appl. Phys. Lett. 75(10), p. 1377-1379 (1999).

3. Ch. Oh, M.J. Eskuti, Time-domain analysis of periodic anisotropic media at oblique incidence: an efficient FDTD implementation // Optics Express 14(24), p. 11870-11884 (2006).

4. C. Deumie', H. Giavannini, C. Amra, Angleresolved ellipsometry of light scattering: discrimination of surface and bulk effects in substrates and optical coatings // Appl. Optics 41(16), p. 3362-3369 (2002).

5. J.-Ch. Chao, W-Y. Wu, A.Y.-G. Fuh, Diffraction characteristics of a liquid crystal polarization grating analysed using the finite-difference timedomain method // Optics Express 15(25), p. 1670216711 (2007).

6. A. Natansohn, B. Kaplan, T. Novikova, A. De Martino, and B. Dre'villon, Characterization of bidimensional gratings by spectroscopic ellipsometry and angle-resolved Muller polarimetry // Appl. Optics 43(6), p. 1233-1240 (2004).

7. T. Clausnitzer, T. Kämpfe, E.-B. Kley, A. Tünnermann, U. Peschel, A.V. Tishchenko and O. Parriaux, An intelligible explanation of highlyefficient diffraction in deep dielectric rectangular transmission gratings // Optics Express 13(26), p. 10448-10456 (2005).
8. O.I. Barchuk, K.S. Bilenko, V.N. Kurashov, Yu.A. Oberemok, S.N. Savenkov, Fine structure of diffraction order polarization characteristics for submicron grating $/ / 8^{\text {th }}$ Ukrainian-Russian seminar “Nanophysics \& Nanoelectronics”, p. 87-88 (2007), Kyiv, Ukraine.

9. O.I. Barchuk, K.S. Bilenko, Yu.A. Oberemok, S.N. Savenkov, Multiple-angle-of-incidence scattering of lossy grating // VIII Intern. Young Scientists Conference on Applied Physics. p. 83-84 (2008), Kyiv, Ukraine.

10. O.I. Barchuk, E.A. Oberemok, T.V. Rodionova, S.N. Savenkov, Polarization characteristics of the polysilicon films structure // Bulletin of the University of Kiev. Physics \& Mathematics 2, p. 211-218 (2007) (in Ukrainian).

11. S.N. Savenkov, V.I. Grygoruk, A.S. Klimov, Ye.A. Oberemok, Yu.A. Skoblya, The method of three input polarizations in Mueller-polarimetry using a polarized source of arbitrary ellipticity // Zhurnal prikladnoy spektroskopii 75(6), p. 875-880 (2008) (in Russian).

12. Y.A. Oberemok, S.N. Savenkov, Solution of the inverse problem of polarimetry for deterministic object on the base of incomplete Mueller matrices // Ukrainian Journal of Physics 47(8), p. 803-807 (2002) (in Ukrainian).

13. S.N. Savenkov, E.A Oberemok, Structure of deterministic Mueller matrices and their reconstruction in the method of three input polarizations // Zhurnal prikladnoy spektroskopii 70(2), p. 224-229 (2003) (in Russian).

14. S.N. Savenkov, E.A. Oberemok, Recovery of the complete Mueller matrix of an arbitrary object in the method of three input polarizations // Zhurnal prikladnoy spektroskopii 71(1), p. 128-132 (2004) (in Russian).

15. V.V. Marienko, B.N. Kolisnichenko, S.N. Savenkov, Parameters optimization of measurement scheme of scattering light matrix // Optika i spektroskopiya 82(5), p. 845-848 (1997) (in Russian).

16. V.V. Marienko, S.N. Savenkov, Representation of arbitrary Mueller matrix in basis of matrix circular and linear anisotropy // Optika i spektroskopiya 76(1), p. 102-104 (1994) (in Russian).

17. S.N. Savenkov, V.V. Marienko, E.A. Oberemok, O. Sydoruk, Generalized matrix equivalence theorem for polarization theory // Phys. Rev. E 74, 056607(8) (2006).

18. C. Russev Stoyan, Drolet Jean-Pierre, and Ducharme Daniel, Standards for which the ellipsometric parameter $\Psi$ remains insensitive to variations in the angle of incidence // Appl. Optics 37(25) p. 5912-5922 (1998). 